\title{
DESGARRO VAGINAL DURANTE EL COITO NORMAL
}

Dr. Jaime Cantillo G.*

Dra. Laura Rojas R.**

\section{Introducción :}

El aumento creciente de los desgarros de paredes vaginales durante el coito nos ha llevado a hacer una revisión sobre el tema, en el Instituto Materno Infantil de Bogotá.

Son muchos los casos publicados por la literatura médica mundial (4); entre nosotros nadie se ha ocupado del tema y hemos visto que solo algunos textos de ginecología dedican unas pocas líneas al problema.

Los desgarros vaginales pueden producirse en:

a) En la desfloración.

b) Coitos violentos.

C) Violaciones.

d) Durante el parto.

e) Por la aplicación de fórceps.

f) Por instrumentos en las maniobras abortivas.

g) Por cuerpos extraños introducidos en la vagina con fines masturbativos, por curiosidad como en las niñas o en aberraciones como suceden en las personas dementes.

h) Por quemaduras cuando se introducen substancias cáusticas con fines antisépticos, como anticonceptivos o para reducir la dilatación vaginal que produce el parto (2).

i) Espontáneamente, en mujeres viejas con atrofia vaginal (4-8).

j) Durante el coito normal con vaginas sanas y sin antecedentes de dispareunia ni de enfermedades ginecológicas (5-6).

En cuanto a la etiopatogenia son muchas las teorías esbozadas pero ninguna es aceptada. Se ha mencionado cierta fragilización por hiperemia de la vagina en el curso del orgasmo. Compresión del aire de la vagina por el pene en erección, el cual actúa a la manera de un pistón.

El prolapso uterino con o sin rectocele, es causa predisponente por aplanamiento de la cúpula vaginal (1). También se ha mencionado, que en el momento del orgasmo se produce un acortamiento perineal con disminución de la altura vaginal, a esto se le agrega la contracción de los músculos de la pared abdominal y del diafragma que aumenta la presión intra abdominal que va a actuar

\footnotetext{
* Instructor Asociado de Anatomía Patológica de la Universidad Nacional, Facultad de Medicina.

* Instructora Asociada de Ginecología y Obstetricia de la Universidad Nacional, Facultad de Medicina.
} 
a nivel del diafragma pélvico, el cual recibe la presión contraria producida por el hombre, determinando un adelgazamiento de las paredes vaginales haciéndolas más frágiles.

En el embarazo se agregan los factores de edema y aumento de vascularización con reblandecimiento de los tejidos, en el puerperio se suma atrofia del epitelio vaginal (7). Todo esto contribuye a que la vagina se haga más débil y en un momento dado pueda romperse con gran facilidad en el momento del coito (1-4).

\section{Material}

Se revisaron 253.249 historias clínicas desde Enero de 1958 a Junio de 1969, del Instituto Materno Infantil de Bogotá; encontramos 40 casos de rupturas vaginales durante el coito normal. Se descartaron todas aquellas pacientes cuya ruptura fue debida a violación, traumatismo o cirugía previa.

Los ingresos por año son los siguientes:

\begin{tabular}{lll}
\hline Años & Ingresos & $\mathbf{N}^{0}$ Casos \\
\hline 1958 & 13.598 & 0 \\
1959 & 15.871 & 0 \\
1960 & 18.254 & 2 \\
1961 & 19.987 & 4 \\
1962 & 21.339 & 3 \\
1963 & 24.198 & 4 \\
1964 & 25.283 & 4 \\
1965 & 26.560 & 2 \\
1966 & 28.510 & 6 \\
1967 & 29.067 & 2 \\
1968 & 28.803 & 6 \\
1969 (Junio) & 15.371 & 7 \\
\hline
\end{tabular}

\section{Resultados}

Podemos observar (ver Tabla N. 1) que en los años 58 y 59 no encon-

TABLA 1

\section{DESGARRO VAGINAL UURANTE EL COITO}

I. $M$.

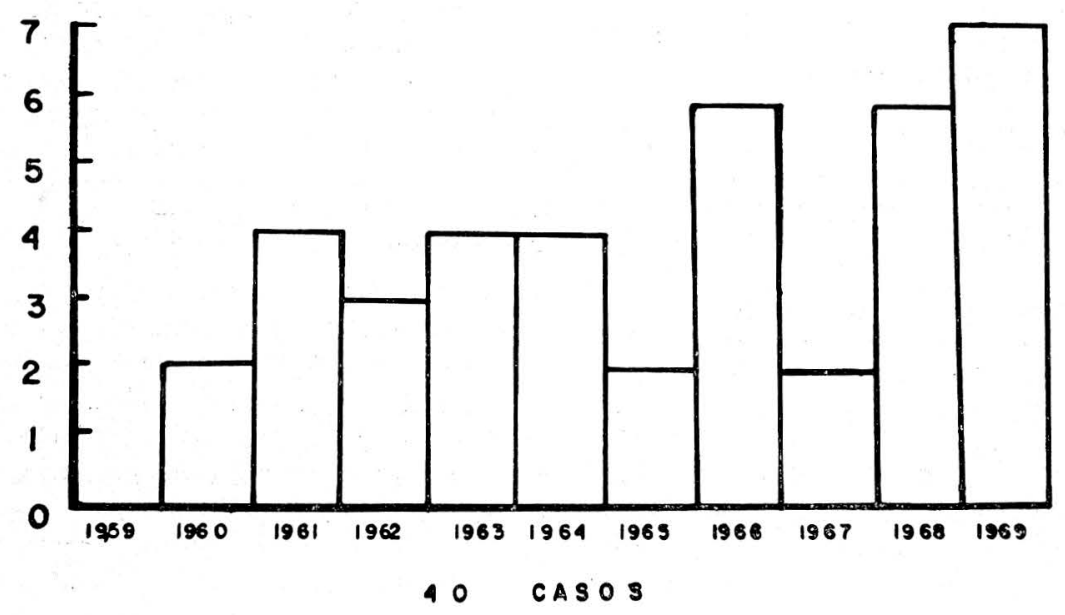


TABLA 2

DESGARRO VAGINAL DURANTE EL COITO

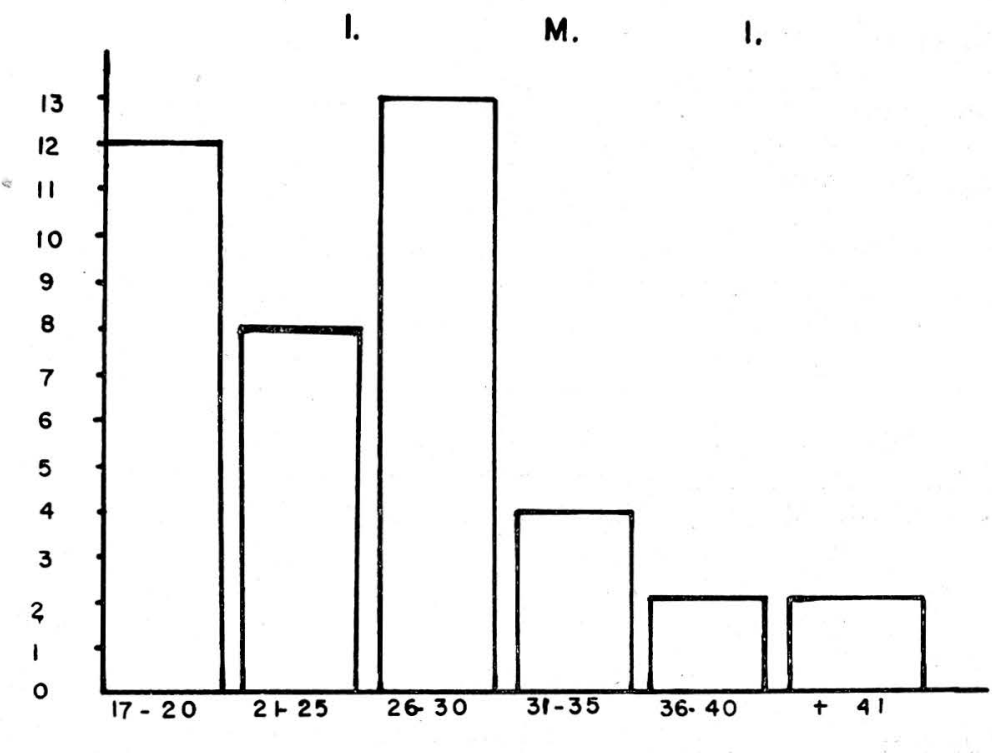

E D A D

tramos ningún caso. El menor número de casos están en los años 60,65 y 67; la mayor incidencia se encuentra en los años 66,68 y en el primer semestre del año 1969, debido talvez a la fusión de los servicios de Ginecología y Obstetricia.

\section{Edad (Ver Tabla № 2}

La edad más joven corresponde a cuatro pacientes de 17 años, sin antecedentes obstétricos. La mayor edad corresponde a una mujer de 45 años, quien había tenido. su último parto normal varios años antes. El mayor número de casos lo encontramos entre las edades de 17 a 20 años, con 12 casos, 8 casos entre los 21 a 25 años y 13 casos entre las edades de 26 a 30 años, resultando un total de 36 casos dentro de la época de mayor actividad reproductora de la mujer.

\section{Paridad (Ver Tabla № 3)}

Como podemos apreciar en la Tabla, la mayor incidencia ocurre en nulíparas y en primíparas con 11 y 9 casos respectivamente, descendiendo la incidencia con la nuliparidad.

\section{Antecedentes ginecológicos y obstétricos (Ver Tabla No 4)}

En 16 pacientes no se pudo determinar antecedentes ginecológicos $u$ obstétricos, porque en las historias clínicas no habían datos concernientes. En 10 pacientes, ellos fueron negativos. Se encontró una paciente de 25 años de edad, G.0 - P.0, quien a los 17 años se le había practicado una traquelectomía por elongatio co- 
TABLA 3

DESGARRO

VAGINAL

DURANTE

EL COITO

I.

M.

I.

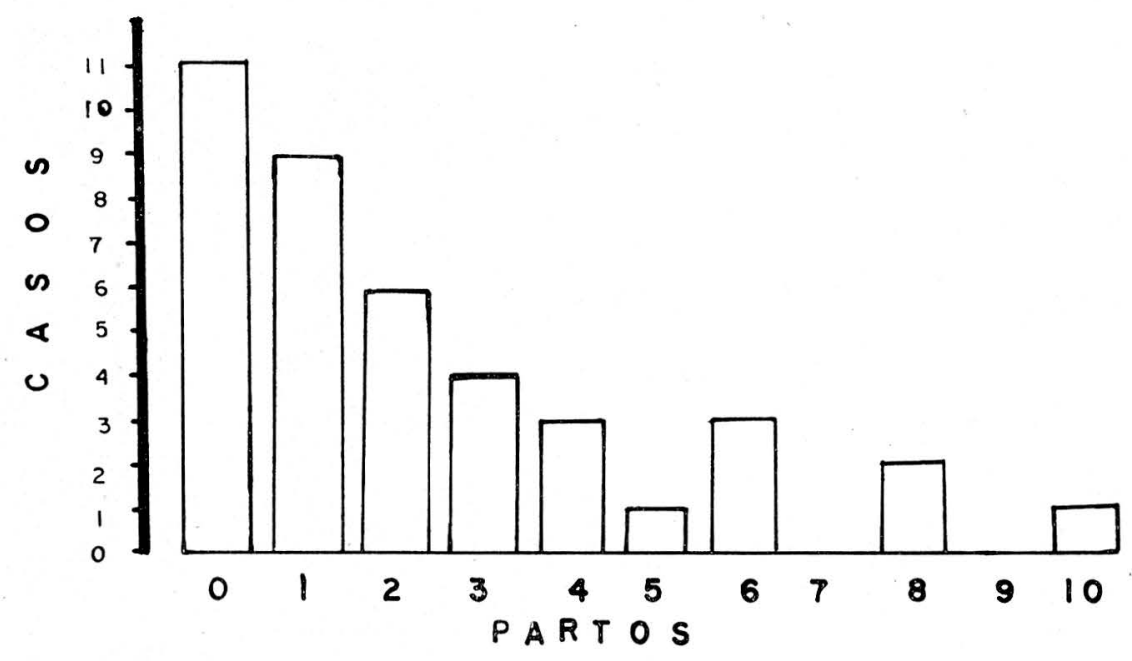

TABLA 4
DESGARRO
VA GINAL
DURANTE
EL COITO
1.
M.
I.

ANTECEDENTES

OBSTETRICOS

GINECOL OGICOS

NEGAT, IV O S

SIN DETERM INAR

TRA QUELECTOMIA

EM B ARAZO

PARTO RECIENTE

LACTANCIA
$10\left(\mathrm{G}_{0} \mathrm{P}_{0}\right)$

16

I (GoPo)

I (2m.)

$6(5-45 d$.

$7(2-4 m)$ 
litis, después del desgarro, el cual se suturó con catgut, tuvo 5 embarazos con partos a término, normales. También encontramos una paciente de 29 años G.3 - P.2, con dos meses de gestación quien ingresó al hospital en estado de shock por anemia aguda debido a un desgarro de fondo de saco posterior, en el sentido transversal de 6 ctms. que no comprometía al peritoneo, se suturó con catgut y evolucionó satisfactoriamente, hubo necesidad de aplicarle sangre para contrarrestar el shock. Vimos una paciente de 29 años de edad G.3 - P.3, quien tuvo el parto en la casa, a los 5 días del puerperio tuvo relaciones sexuales que le produjeron un desgarro de 8 ctms., localizado en el fondo de saco posterior. 7 mujeres estaban lactando, el tiempo de lactancia osciló entre 2 y 4 meses.
Localización, tratamiento y evolución (Ver Tabla No 5)

Los desgarros vaginales durante el coito, como vemos en la gráfica pueden sucederse en cualquier sitio de las paredes vaginales. Pero la mayor incidencia la pudimos observar en la pared posterior con 21 casos y en orden de frecuencia sigue la derecha con 10 y la izquierda con 5 casos. En 2 no se pudo determinar el sitio, por falta de datos en la historia clínica. De estos últimos, uno corresponde a una mujer de 17 años de edad G.0 - P.O, sin antecedentes ginecológicos $\mathrm{U}$ obstétricos, ingresó al IMI en shock debido a hemorragia profusa por vagina. Se trató con sangre y taponamiento. El otro corresponde a una mujer de 40 años $G .5$ P.5, quien dos meses antes había te-

TABLA 5

$\begin{array}{ccccc}\text { DESGARRO } & \text { VAGINAL } & \text { DURANTE } & \text { COL } & \text { COITO } \\ & \text { I. } & \text { M. } & \text { I. } \\ \text { LOCALIZACION } & \text { CASOS } & \text { LONGITUD TRATAMIENTO EVOLUCION }\end{array}$

AN TERIOR

POSTERIOR

DERE CHA

IZQUIERDA

SIN DATO
2

21

10

5

$8 \mathrm{~cm}$.

MINIMA

$2 \mathrm{~cm}$.
S UT UR A

34

$S$

A

I

$s$

MAXIMA

OBSERVACION

5

A

C

$\mathrm{T}$

0

R

HISTERECTOMIA । 
nido un parto intervenido con cesárea en la misma Institución, el desgarro se suturó y salió del hospital sin complicaciones. 34 pacientes se trataron con sutura, cinco con taponamientos y observación. Todas las pacientes evolucionaron satisfactoriamente sin ninguna complicación.

Una paciente (Historia Clínica № 187.779), motivo de esta revisión, terminó en histerectomía, por diagnóstico errado. Ella era una mujer de 30 años, puérpera de 20 días, quien había tenido un parto en pelvis atendido en esta Institución y a quien se le practicó revisión uterina por hemorragia profusa durante el alumbramiento, 20 días después, ingresa nuevamente con hemorragia abundante de 1 hora de evolución. Se hace diagnóstico de ruptura uterina cérvicosegmentaria, por lo cual se lleva a laparotomía en estado de shock. En el acto operatorio observaron sangre libre en cavidad (5 Occ.) y un hematoma del ligamento ancho izquierdo con una solución de continuidad que iba por todo el borde uterino, cuello y parte superior de vagina, motivo por el cual se practicó histerectomía total. El examen anatomopatológico, protocolo № $1570-69$ reportó un útero normal. La paciente dio como antecedente de importancia que la hemorragia se había iniciado después del coito y el caso se interpretó finalmente como un desgarro de fondo de saco posterior con ruptura del peritoneo y formación de hematoma en el ligamento ancho el cual se confundió con ruptura uterina.

\section{Discusión}

Los 40 casos de rupturas de las paredes vaginales durante el coito normal corresponden a mujeres ginecológicamente sanas; sin este incidente en sus relaciones sexuales anteriores.
Se desecharon los casos de rupturas vaginales producidas en la desfloración, violación, traumatismo como puntapiés en las regiones genitales o caídas a horcajadas, estrechez vaginal por cualquier causa y aquellas a quienes se les había realizado cirugía por cualquier motivo y que ella hubiese dejado alguna secuela post-quirúrgica en la vagina, por ese motivo incluímos el caso de la traquelectomía que no lesionó en lo más mínimo las paredes vaginales.

Los desgarros vaginales pueden ocurrir en cualquier edad (1-5). En nuestra Institución la curva se hizo más alta en las mujeres cuya edad osciló entre los 17 a 30 años de edad, mostrando 33 casos con un porcentaje del $82,5 \%$. Las rupturas de las paredes vaginales en mujeres viejas pueden ocurrir espontáneamente como se ve en los tres casos relatados por Purnell (8) y se observa claramente en el caso publicado por Morrison (4), que corresponde a una mujer de 61 años de edad a quien por un estornudo se le produjo un desgaro vaginal que debió suturarse. De las 253.249 historias clínicas revisadas no encontramos ningún caso de rupturas vaginales espontáneas por atrofia vaginal como en los casos anteriormente citados. En estos $40 \mathrm{ca}$ sos también se observa una gran elevación de la curva en las nulíparas y primíparas con el $27,5 \%$ y $22,5 \%$ respectivamente, descendiendo la curva con la multiparidad, ella lleva consigo una dilatación vaginal, por tal motivo, entre los árabes las estrecheces vaginales, la criptomenorrea y otros problemas ginecológicos son frecuentes, porque las mujeres por el temor de perder el marido se colocan dentro de la vagina sustancias como la sal de roca para reducir la dilatación vaginal, lográndola hacer más estrecha y poder así mantener siempre 
a su lado a los esposos (2). Sloan (9) relata el caso de una mujer de 33 años de edad, quien sufrió ruptura del cuerpo amarillo del embarazo durante el coito con el consecutivo aborto sin ruptura de las paredes vaginales. Dentro del material examinado no encontramos ningún caso similar, pero sí observamos un caso de ruptura peritoneal que terminó en histerectomía (6) y otro de ruptura peritoneal después de 20 meses de histerectomía (10). La literatura confirma el dato de que las rupturas vaginales durante el coito son frecuentes en el puerperio y la lactancia por atrofia vaginal (1-7).

\section{Resumen}

Se revisaron las historias clínicas del I.M.I. de Bogotá durante un período de $11 \frac{1}{2}$ años, encontrando 40 casos de ruptura vaginal imputable al coito normal. Se hace una revisión de la literatura y se explican sus posibles etiologías. En el análisis de edad, paridad, antecedentes obstétricos ginecológicos de las pacientes, encontramos que el accidente es más frecuente en nuligestantes o en mujeres con un solo hijo, la edad oscila entre los 26 a 20 años pudiéndose presentar a cualquier edad una vez iniciada la vida sexual. El parto y la lactancia son factores predisponentes. La localización más frecuente es en pared posterior de vagina, el tratamiento casi siempre es quirúrgico de acuerdo a la longitud del desgarro, presentamos un caso en el que se practicó histerectomía abdominal por ruptura del fondo de saco peritoneal posterior el cual se confundió con ruptura uterina debido al antecedente de un parto reciente. Todos los casos evolucionaron satisfactoriamente.

\section{Summary}

Forty cases of vaginal rupture due to normal coitus were found among the clinical histories at Bogotá's Instituto Materno Infantil, in an eleven and a half years' period. The authors review the literature and explain the different etiologies possible. Analyzing age, deliveries and gynecological and obstetrical history of the patients, they found that the accident is more frequent in women without children, or with just one; the age fluctuates between twenty and twenty-six, occuring at any age after initiation of sexual life. Delivery and nursing are predisponing factors. The most frequent localization is the vagina's posterior wall, the treatment is almost always surgical, depending on the lenght of the laceration. A case is presented in which andabdominal histerectomy was necessary due to rupture of the posterior peritoneal membrane, that was mistaken for an uterine rupture, due to a recent delivery. All cases had a satisfactory evolution.

\section{BIBLIOGRAFIA}

1 BURDMAN, M.: Vaginal injury at coitus. Brit. M. J., 2: 226-227, 1948.

2 L. J. GERMAN: Some Obstetrical aspects of salt induced vaginal stenosis in Bahrain: J. Obstet. Gynec. Brit. Comm: 75-674-7, june 1968.

3 LOWRIE ROBERT J. CHARLES C. THOMAS. Injuries of the vagina ginecology Illinois, U.S.A. First Edition 1952.

4 MORRISON, J.: Vault Laceration due to sneezing. J. Obst. Gynaec Brit. Comm. 74 : 773, 1967.

5 NICHOLLS, R. B.: Ruptura of the posterior vagina during normal coitus. Amer. J. Obstet. Gynec. 52: 500, 1946.

6 PUDLIK KOLACZKOWSCA C.: A case of vaginal wall injury during coitus with perfo- 
ration into the peritoneal cavity. Ginek. Pol. 37: 1235-7, 1966.

7 J. P. PUNDEL: Colpocitología hormonal. Torey-Masson. Ed. Barcelona - España, 1968.

8 PURNELL, L. W. Spontaneons Rupture of the vaginal vault. J. Obst. Gynec. Comm. 72: 799-801, 1965.
9 SLOAN, W. R.: Rupture of corpus luteum of pregnancy during coitus: Brit. med. J. № $4565: 26,1948$.

10 WESSEY et al: Traumatic ruptura of the apex of the vagina. Twenty months following abdominal histerectomy. Am. J. Obst. Gynec: 76: 1207, 1958. 